\title{
A Hull and White Formula for a General Stochastic Volatility Jump-Diffusion Model with Applications to the Study of the Short-Time Behavior of the Implied Volatility
}

\author{
Elisa Alòs, ${ }^{1}$ Jorge A. León, ${ }^{2}$ Monique Pontier, ${ }^{3}$ and Josep Vives ${ }^{4}$ \\ ${ }^{1}$ Departament d'Economia i Empresa, Universitat Pompeu Fabra, Ramon Trias Fargas 25-27, \\ 08005 Barcelona, Spain \\ 2 Departamento de Control Automático, Centro de Investigación y de Estudios Avanzados del Instituto \\ Politécnico Nacional (CINVESTAV-IPN), Apartado Postal 14-740, CP 07000 México D.F., Mexico \\ ${ }^{3}$ Institut Mathématique de Toulouse, Université de Toulouse, 31062 Toulouse cedex 9, France \\ ${ }^{4}$ Departament de Probabilitat, Lògica i Estadística, Universitat de Barcelona, \\ Gran Via de les Corts Catalanes 585, 08007 Barcelona, Spain
}

Correspondence should be addressed to Josep Vives, josep.vives@ub.edu

Received 1 April 2008; Revised 3 September 2008; Accepted 25 November 2008

Recommended by Wenbo Li

\begin{abstract}
We obtain a Hull and White type formula for a general jump-diffusion stochastic volatility model, where the involved stochastic volatility process is correlated not only with the Brownian motion driving the asset price but also with the asset price jumps. Towards this end, we establish an anticipative Itô's formula, using Malliavin calculus techniques for Lévy processes on the canonical space. As an application, we show that the dependence of the volatility process on the asset price jumps has no effect on the short-time behavior of the at-the-money implied volatility skew.
\end{abstract}

Copyright (C) 2008 Elisa Alòs et al. This is an open access article distributed under the Creative Commons Attribution License, which permits unrestricted use, distribution, and reproduction in any medium, provided the original work is properly cited.

\section{Introduction}

It is well known that classical stochastic volatility models, where the volatility is allowed to be a diffusion process, are able to capture the dependence of the implied volatility as a function of the strike (the smile or the skew). Nevertheless, they can not explain its dependence with respect to time to maturity (term structure). For example, empirical observations indicate that the at-the-money skew slope is approximately $O\left((T-t)^{-1 / 2}\right)$ (here $T-t$ denotes the time to maturity), while the rate for these stochastic volatility models is $O(1)$ (e.g., Lewis [1], Lee [2], or Medvedev and Scaillet [3]). The introduction of jumps in the asset price dynamics is a natural extension of classical stochastic volatility models proposed with the aim to capture 
this short-time behavior. Although the rate of the skew slope for models with jumps is still $O(1)$ (see Medvedev and Scaillet [3]), they allow flexible modelling, and generate skews and smiles similar to those observed empirically (see Bates [4], Barndorff-Nielsen and Shephard $[5,6]$, or Carr and $\mathrm{Wu}[7])$.

In Alòs et al. [8], the authors considered general jump-diffusion stochastic volatility models where the volatility is not necessarily a diffusion. They proved that for a volatility process independent of price jumps (as in the Bates [4] case) the at-the-money skew slope explodes if and only if the Malliavin derivative of the volatility process $D_{s} \sigma_{t}$ also does when $s \uparrow t$. This allows us to consider new models-where the volatility is not required to be Markovian nor to be a diffusion process-that capture the short-time explosion of skew slopes. The basic idea in that work was to expand option prices around the classical Hull and White expression by means of Malliavin calculus, following similar ideas as in Alòs [9]. This gives us a decomposition of option prices that allows us to identify the effect of correlation (between the volatility and the Brownian motion driving stock prices) and the effect of jumps in the at-the-money skew slope when time to maturity tends to zero. This can be interpreted as an answer of a demand in Fouque et al. [10, end of page 54].

In this paper, we study jump-diffusion stochastic volatility models allowing the volatility process to be correlated also with the price jumps (see Bakshi et al. [11] and Duffie et al. [12], among others). Our main goal in this work is to describe analytically the effect of this extension in the at-the-money short-time behavior of the stochastic volatility. The idea will be again to decompose option prices around the Hull and White term, now by using Malliavin calculus for Lévy processes (see Solé et al. [13], Løkka [14] and Petrou [15]). In comparison with the formula obtained in Alòs et al. [8], here we obtain an extra term because the volatility depends now on the jump price. This representation allows us to show that the existence of correlation between the volatility process and the price jumps does not have any influence on the at-the-money skew of the implied volatility as time runs to expiry, confirming an heuristic idea explained in Gatheral [16, page 70].

The paper is organized as follows. In Section 2, we give the main hypotheses and notations. Section 3 is devoted to introduce the Malliavin calculus framework needed in the remaining of the paper. In Section 4, we obtain the Hull and White formula. In Section 5, we apply it to the problem of describing the at-the-money short time skew of the implied volatility. Finally, Section 6 is devoted to the conclusions.

\section{Main hypotheses and notations}

We consider a log-price process, under the market chosen risk-neutral probability measure, given by

$$
X_{t}=x+(r-\lambda k) t-\frac{1}{2} \int_{0}^{t} \sigma_{s}^{2} d s+\int_{0}^{t} \sigma_{s}\left(\rho d W_{s}+\sqrt{1-\rho^{2}} d B_{s}\right)+Z_{t}
$$

where, $t \in[0, T], x$ is the current log-price, $r$ is the instantaneous interest rate, $W$ and $B$ are independent standard Brownian motions, $\rho \in[-1,1]$, and $Z$ is a compound Poisson process, independent of $W$ and $B$, with intensity $\lambda$, finite Lévy measure $v$, and with $k:=(1 / \lambda) \int_{\mathbb{R}}\left(e^{y}-\right.$ 1) $v(d y)$.

We assume that the process $\sigma$ is adapted to the filtration generated by $W$ and $Z$. So, in this paper, generalizing Alòs et al. [8], we allow the volatility to have nonpredictable jump times as advocated by Bakshi et al. [11] and Duffie et al. [12], among others. 
In the following, we denote by $\mathcal{F}^{W}, \Psi^{B}$, and $\mp^{Z}$ the filtrations generated by the independent processes $W, B$, and $Z$, respectively. Moreover, we define $\mathcal{F}:=\mathcal{F}^{W} \vee \mathcal{F}^{B} \vee \mathcal{F}^{Z}$.

It is well known that if we price a European call with strike price $K$ by the formula

$$
V_{t}=e^{-r(T-t)} E\left[\left(e^{X_{T}}-K\right)_{+} \mid \mathcal{F}_{t}\right]
$$

where $E$ denotes the expectation with respect to a risk-neutral measure, there is no arbitrage opportunity. Thus $V_{t}$ is a possible price for this derivative.

In the sequel, we use the following notation:

(i) The process $v_{t}:=\left(Y_{t} /(T-t)\right)^{1 / 2}$, with $Y_{t}:=\int_{t}^{T} \sigma_{s}^{2} d s$, denotes the future average volatility.

(ii) With $\mathrm{BS}(t, x, \sigma)$ we represent the classical Black-Scholes function with constant volatility $\sigma$, current $\log$ stock price $x$, time to maturity $T-t$, strike price $K$, and interest rate $r$. This function can be written as

$$
\mathrm{BS}(t, x, \sigma)=e^{x} \Phi\left(\frac{x-x_{t}^{*}}{\sigma \sqrt{T-t}}+\frac{\sigma}{2} \sqrt{T-t}\right)-e^{x_{t}^{*}} \Phi\left(\frac{x-x_{t}^{*}}{\sigma \sqrt{T-t}}-\frac{\sigma}{2} \sqrt{T-t}\right),
$$

where $x_{t}^{*}=\log K-r(T-t)$ is the future log-price at $t$ and $\Phi$ is the cumulative probability function of the standard normal law.

(iii) With $N$ we denote the Poisson random measure on $[0, T] \times \mathbb{R}$ such that $Z_{t}=$ $\int_{[0, t] \times \mathbb{R}} x N(d s, d x)$. Moreover, $\widetilde{N}(d s, d x):=N(d s, d x)-d s v(d x)$ is the compensated Poisson random measure.

(iv) We consider the operator $\mathcal{L}_{\mathrm{BS}}(\sigma):=\partial_{t}+(1 / 2) \sigma^{2} \partial_{x x}^{2}+\left(r-(1 / 2) \sigma^{2}\right) \partial_{x}-r$ which satisfies $\mathcal{L}_{\mathrm{BS}}(\sigma) \mathrm{BS}(\cdot, \cdot, \sigma)=0$.

\section{Required tools of Malliavin calculus for Lévy processes}

\subsection{Introduction}

In this section, we introduce the tools of Malliavin calculus for Lévy processes that we need in the rest of the paper.

Consider a complete probability space $(\Omega, \mathcal{F}, \mathbb{P})$ and let $L=\left\{L_{t}, t \in[0, T]\right\}$ be a càdlàg Lévy process with triplet $(\gamma, \sigma, v)$. See for example the book of Sato [17] for a general theory of Lévy processes.

It is well known that $L$ can be represented as

$$
L_{t}=\gamma t+\sigma W_{t}+\iint_{(0, t] \times\{|x|>1\}} x N(d s, d x)+\lim _{\epsilon \downarrow 0} \iint_{(0, t] \times\{\epsilon<|x| \leq 1\}} x \widetilde{N}(d s, d x),
$$

where $W$ is a Brownian motion and $N$ is the Poisson random measure associated to $v$. It is also known that $\mp^{L}=\mp^{W} \vee \Psi^{N}$. See for example, Solé et al. [13].

In general, the construction of a Malliavin calculus, based on a chaos expansion, for a certain process follows three main steps. First of all, to prove a chaotic representation 
property, secondly, to define formally the gradient and divergence operators, and finally, to give their probabilistic interpretations.

In the last years, several approaches to the Malliavin calculus for Lévy processes have been developed, with different probabilistic interpretations of gradient operators. Between them, we mention the approach of Nualart and Schoutens [18], the approach of Sole et al. [13], based on Itô [19], and the approach of Løkka [14] and Petrou [15]. In this paper, we follow the last one, because in it, the form of the gradient operator simplifies strongly our computations. As a tool for our results we develop in Sections 3.3 and 3.4 two transfer formulas between the second and the third methods. Finally, let us remark that we are under the conditions of Løkka's approach because our Lévy measure $v$ is finite.

\subsection{The chaotic representation property}

Consider the space $[0, T] \times \mathbb{R}$ with its Borel family of sets $\mathbb{B}([0, T] \times \mathbb{R})$. We can introduce the centered independent random measure given by

$$
M(E):=\sigma \int_{E(0)} d W_{t}+\iint_{E^{\prime}} \widetilde{N}(d t, d x), \quad E \in \mathcal{B}([0, T] \times \mathbb{R}),
$$

where $E(0):=\{t \in[0, T]:(t, 0) \in E\}$ and $E^{\prime}:=E-E(0) \times\{0\}$. Its variance is given by

$$
\mu(E)=\sigma^{2} \int_{E(0)} d t+\iint_{E^{\prime}} d t v(d x)
$$

Note that $W$ can be seen as a centered independent Gaussian random measure on $[0, T]$, and $\widetilde{N}(d s, d x)$ can be seen as a centered independent random measure on $[0, T] \times \mathbb{R}_{0}$ where $\mathbb{R}_{0}=\mathbb{R}-\{0\}$. Thus we can write

$$
M(d s, d x)=\sigma\left(W \otimes \delta_{0}\right)(d s, d x)+\widetilde{N}(d s, d x)
$$

where $\delta_{0}$ is the Dirac's delta, that is, a unitary mass on the point $\{0\}$.

In this context, we can define stochastic multiple integrals $I_{n}$ with respect to $M$ with kernels in the Hilbert spaces

$$
\mathbb{L}_{n}^{2}:=L^{2}\left(([0, T] \times \mathbb{R})^{n}, \mathbb{B}([0, T] \times \mathbb{R})^{n}, \mu^{\otimes n}\right),
$$

in the usual way, and to prove that if $\left\{\mathcal{F}_{t}^{X}, t \in[0, T]\right\}$ is the completed natural filtration of $X$, for any random variable $F \in L^{2}\left(\Omega, \Psi_{T}^{X}, \mathbb{P}\right)$ we have the chaotic representation

$$
F=\sum_{n=0}^{\infty} I_{n}\left(f_{n}\right)
$$

where the kernels are unique if we take them symmetric. 


\subsection{The Malliavin-type derivative}

Let us denote by $\mathbb{D}^{1,2}$ the set of random variables in $L^{2}(\Omega)$, of the form (3.6), such that $\sum_{n=1}^{\infty} n n !\left\|f_{n}\right\|_{L_{n}^{2}}^{2}<\infty$. The Malliavin derivative $D^{M} F$ of a random variable $F \in \mathbb{D}^{1,2}$ is the process $\left\{D_{t, x}^{M} F,(t, x) \in[0, T] \times \mathbb{R}\right\}$ defined by $D_{t, x}^{M} F:=\sum_{n=1}^{\infty} n I_{n-1}\left(f_{n}((t, x), \cdot)\right)$.

In order to give the probabilistic interpretation of this operator, we assume, in the remaining, as in Solé et al. [13], that the underlying probability space is the canonical Lévy space $\left(\Omega^{W} \times \Omega^{N}, \mathcal{F}^{W} \otimes \mathcal{F}^{N}, \mathbb{P}^{W} \otimes \mathbb{P}^{N}\right)$. That is, $\left(\Omega^{W}, \mathcal{F}^{W}, \mathbb{P}^{W}\right)$ is the canonical Wiener space and $\left(\Omega^{N}, \mathscr{F}^{N}, \mathbb{P}^{N}\right)$ is the canonical Lévy space of the compound Poisson process with Lévy measure $v$. Moreover, we assume that $W$ and $Z$ are the canonical processes.

Let $\omega=\left(\omega^{W}, \omega^{N}\right)$ be an element of this space. So, $\omega^{W}$ is a continuous trajectory null at the origin and $\omega^{N}$ is a sequence of (jump instant, jump size) pairs

$$
\omega^{N}:=\left(\left(t_{1}, x_{1}\right),\left(t_{2}, x_{2}\right),\left(t_{3}, x_{3}\right), \ldots\right) .
$$

From Petrou [15], we have

$$
D_{t, 0}^{M}=\frac{1}{\sigma} D_{t}^{W} \mathbb{1}_{\{\sigma>0\}},
$$

where $D_{t}^{W}$ denotes the classical Malliavin derivative with respect to the Brownian motion $W$ (e.g., Nualart [20]). Denote by $\mathbb{D}^{W}$ its domain.

In order to obtain the probabilistic interpretation of $D_{t, x}^{M}$ for $x \neq 0$, we consider the following transformation.

Given $(t, x) \in[0, T] \times \mathbb{R}_{0}$, we can add to any $\omega^{N}$ a jump of size $x$ at instant $t$, denote the new element

$$
\omega_{t, x}^{N}:=\left((t, x),\left(t_{1}, x_{1}\right),\left(t_{2}, x_{2}\right), \ldots\right)
$$

and write $\omega_{t, x}:=\left(\omega^{W}, \omega_{t, x}^{N}\right)$. So, for any $(t, x) \in[0, T] \times \mathbb{R}_{0}$, we can define the operator $T_{t, x} F:=$ $F\left(\omega_{t, x}\right)$. As it is shown in Sole et al. [13, Proposition 4.8] this is a well-defined operator.

For all $F \in \mathbb{D}^{1,2}$, define

$$
D_{t, x}^{N} F=T_{t, x} F-F, \quad x \neq 0
$$

and denote by $\mathbb{D}^{N}$ its domain.

This operator is related to $D^{M}$. Indeed, in Solé et al. [13] is considered the random measure

$$
\bar{M}(E):=\sigma \int_{E(0)} d W_{t}+\iint_{E^{\prime}} x d \widetilde{N}(t, x), \quad E \in B([0, T] \times \mathbb{R}) .
$$

Observe that

$$
\bar{M}(E)=I_{1}\left(h(x) \mathbb{1}_{E}(t, x)\right),
$$


where

$$
h(x):=x \mathbb{1}_{\{x \neq 0\}}+\mathbb{1}_{\{x=0\}} .
$$

Therefore, we obtain the transfer principle

$$
D_{t, x}^{M} F=h(x) D_{t, x}^{\bar{M}} F
$$

for $F \in \mathbb{D}^{1,2}$ and $(t, x) \in[0, T] \times \mathbb{R}$. Here $D^{\bar{M}}$ is the annihilation operator used in Solé et al. [13].

Thus, combining results from Solé et al. [13] and Alòs et al. [21] it is easy to show that for $F \in L^{2}(\Omega)$,

$$
D^{N} F \in L^{2}\left([0, T] \times \mathbb{R}_{0}, \mu\right), \quad F \in \mathbb{D}^{W} \Longleftrightarrow F \in \mathbb{D}^{1,2},
$$

and in this case $D_{t, x}^{M} F=D_{t, x}^{N} F, x \neq 0$.

Observe that we have proved

$$
D_{t, x}^{M}=\mathbb{1}_{\{\sigma>0\}} \mathbb{1}_{\{0\}}(x) \frac{1}{\sigma} D_{t}^{W}+\mathbb{1}_{\mathbb{R}_{0}}(x) D_{t, x}^{N}
$$

which follows from (3.8) and (3.10).

Observe also that it is immediate from (3.10), to see that

$$
D_{t, x}^{N}(F G)=F D_{t, x}^{N} G+G D_{t, x}^{N} F+D_{t, x}^{N} F D_{t, x}^{N} G
$$

Finally, we have $\mathbb{D}^{1,2}=\mathbb{D}^{W} \cap \mathbb{D}^{N}$, due to Solé et al. [13, Section 2] and the equalities (3.12) and (3.14).

\subsection{The Skorohod-type integral}

Let $u \in L^{2}(\Omega \times[0, T] \times \mathbb{R}, \mathbb{P} \otimes \mu)$. For almost all $(t, x)$ we have the chaotic decomposition (see Section 3.2)

$$
u_{t, x}=\sum_{n=0}^{\infty} I_{n}\left(f_{n}(t, x, \cdot)\right)
$$

where $f_{n} \in \mathbb{L}_{n+1}^{2}$ is symmetric in the $n$ last variables.

Let $\widehat{f}_{n}$ be the symmetrization in all $n+1$ variables. Then, we define the Skorohod integral of $u$ by

$$
\delta(u)=\sum_{n=0}^{\infty} I_{n+1}\left(\widehat{f}_{n}\right),
$$


in $L^{2}(\Omega)$, provided $u \in \operatorname{Dom} \delta^{M}$, that means

$$
\sum_{n=0}^{\infty}(n+1) !\left\|\widehat{f}_{n}\right\|_{L_{n+1}^{2}}^{2}<\infty
$$

Moreover, if $F \in \mathbb{D}^{1,2}$ and $u \in \operatorname{Dom} \delta^{M}$ we have the duality relation

$$
E\left(F \delta^{M}(u)\right)=E \int_{0}^{T} \int_{\mathbb{R}} u(t, x) D_{t, x}^{M} F \mu(d t, d x)
$$

So, in this sense, $\delta^{M}$ is the dual of the operator $D^{M}$. Sometimes we will write $\delta_{t}(u)$ instead of $\delta(u \mathbb{1}[0, t])$.

Note, also, that the transfer principle (3.14) allows us to establish a transfer principle between $\delta^{M}$ and the divergence operator $\delta^{\bar{M}}$ given in Solé et al. [13]. Namely,

$$
\delta^{M}(u)=\delta^{\bar{M}}\left(\frac{u}{h}\right), \quad u \in \operatorname{Dom} \delta^{M}
$$

The following lemma is useful for our purposes. A version of this lemma in the pure jump case is given in Di Nunno et al. [22, Theorem 3.13].

Lemma 3.1. Let $F \in \mathbb{D}^{1,2}$ and $u \in \operatorname{Dom} \delta^{M}$ such that $u \cdot\left(F+D^{M} F \cdot \mathbb{1}_{\mathbb{R}_{0}}\right) \in L^{2}(\Omega \times[0, T] \times \mathbb{R}, \mathbb{P} \otimes \mu)$. Then,

$$
u \cdot\left(F+D^{M} F \cdot \mathbb{1}_{\mathbb{R}_{0}}\right) \in \operatorname{Dom}^{M} \Longleftrightarrow F \delta^{M}(u)-\int_{[0, T] \times \mathbb{R}} u(t, x) D_{t, x}^{M} F \mu(d t, d x) \in L^{2}(\Omega),
$$

and in this case

$$
\delta^{M}(u \cdot F)=F \delta^{M}(u)-\delta^{M}\left(u \cdot D^{M} F \cdot \mathbb{1}_{\mathbb{R}_{0}}\right)-\int_{[0, T] \times \mathbb{R}} u(t, x) D_{t, x}^{M} F \mu(d t, d x) .
$$

Proof. This result follows using relations (3.16) and (3.21); Alòs et al. [21, Lemma 2.4 and Proposition 2.5] and the transfer formulas (3.14) and (3.22). Note that, in our case, $F$ does not need to be bounded. This is a consequence of the fact that if $G$ is a bounded random variable of $L^{2}\left(\Omega^{N}\right)$ and $v$ is finite, we have that $G \in \mathbb{D}^{N}$ and $D^{N} G$ is also bounded.

In order to give the relation between $\delta^{M}$ and the pathwise integral with respect to $N$, we consider the following two sets.

Definition 3.2. We define $\mathbb{L}^{1,2}:=L^{2}\left([0, T] \times \mathbb{R} ; \mathbb{D}^{1,2}\right)$.

Observe that if $u=\{u(s, y):(s, y) \in[0, T] \times \mathbb{R}\}$ is a random field of $\mathbb{L}^{1,2}$ we have, in particular, that $u$ and $D^{M} u$ are in $L^{2}(\mathbb{P} \otimes \mu)$ and $L^{2}(\mathbb{P} \otimes \mu \otimes \mu)$, respectively. Moreover $\mathbb{L}^{1,2} \subseteq \operatorname{Dom} \delta^{M}$. 
Definition 3.3. We define $\mathbb{L}_{-}^{1,2}$ as the subset of $\mathbb{L}^{1,2}$ of random fields $u$ such that the following $\mathbb{P} \otimes \mu$-a.s. left-limits exist and belong to $L^{2}(\mathbb{P} \otimes \mu)$

$$
\begin{aligned}
u^{-}(s, y) & =\lim _{r \uparrow s, x \uparrow y} u(r, x), \\
D^{-} u(s, y) & =\lim _{r \uparrow s, x \uparrow y} D_{s, y}^{M} u(r, x) .
\end{aligned}
$$

Proposition 3.4. Assume that $u$ is a random field belonging to $\mathbb{L}_{-}^{1,2}$. Assume

$$
\int_{0}^{T} \int_{\mathbb{R}_{0}}\left|u^{-}(s, x)\right| N(d s, d x) \in L^{2}(\Omega)
$$

where $\int_{0}^{T} \int_{\mathbb{R}_{0}} u(s, x) N(d s, d x)$ is the classical path-by-path integral defined by $\sum_{\Delta Z_{t} \neq 0} u\left(t, \Delta Z_{t}\right)$.

Then, $T^{-} u:=u^{-}+D^{-} u \in \operatorname{Dom} \delta^{M}$, and in this case,

$$
\delta^{M}\left(\left(u^{-}+D^{-} u\right) \cdot \mathbb{1}_{\mathbb{R}_{0}}\right)=\int_{0}^{T} \int_{\mathbb{R}_{0}} u^{-}(s, x) \widetilde{N}(d s, d x)-\int_{0}^{T} \int_{\mathbb{R}_{0}} D^{-} u(s, x) \mathcal{v}(d x) d s,
$$

or equivalently,

$$
\delta^{M}\left(T^{-} u \cdot \mathbb{1}_{\mathbb{R}_{0}}\right)=\int_{0}^{T} \int_{\mathbb{R}_{0}} u^{-}(s, x) N(d s, d x)-\int_{0}^{T} \int_{\mathbb{R}_{0}} T^{-} u(s, x) v(d x) d s .
$$

Proof. Assume as a first step that $u \in \mathbb{L}_{-}^{1,2}$ is bounded. Then, $D^{M} u, u^{-}$and $D^{-} u$ are also bounded on $\mathbb{R}_{0}$. In particular (3.26) is true.

We begin considering the following partition of $[0, \infty) \times \mathbb{R}$ :

$$
\begin{gathered}
0=s_{0}<s_{1}<\cdots<s_{n}<\infty=s_{n+1}, \\
-\infty=x_{0}<x_{1}<\cdots<x_{m}<\infty=x_{m+1} .
\end{gathered}
$$

Then, we can define

$$
u^{n, m}(s, x)=\sum_{i=0}^{n} \sum_{j=0}^{m} u\left(s_{i}, x_{j}\right) \mathbb{1}_{\left(s_{i}, s_{i+1}\right]}(s) \mathbb{1}_{\left(x_{j}, x_{j+1}\right]}(x) .
$$


Using Lemma 3.1, we have that for all $n$ and $m$

$$
\begin{aligned}
\delta^{M}\left(u^{n, m} \cdot \mathbb{1}_{\mathbb{R}_{0}}\right)+\delta^{M}\left(D u^{n, m} \cdot \mathbb{1}_{\mathbb{R}_{0}}\right) \\
=\sum_{i=0}^{n} \sum_{j=0}^{m} u\left(s_{i}, x_{j}\right) \delta^{M}\left(\mathbb{1}_{\left(s_{i}, s_{i+1}\right]} \mathbb{1}_{\left(x_{j}, x_{j+1}\right]} \mathbb{1}_{\mathbb{R}_{0}}\right) \\
\quad-\sum_{i=0}^{n} \sum_{j=0}^{m} \int_{0}^{T} \int_{\mathbb{R}_{0}} \mathbb{1}_{\left(s_{i}, s_{i+1}\right]}(s) \mathbb{1}_{\left(x_{j}, x_{j+1}\right]}(x) D_{s, x}^{M} u\left(s_{i}, x_{j}\right) v(d x) d s .
\end{aligned}
$$

First of all, observe that if $\left.r, s \in] s_{i}, s_{i+1}\right]$ and $\left.\left.x, y \in\right] x_{j}, x_{j+1}\right]$, then $\left(D_{s, y}^{M} u^{n, m}\right)(r, x)=$ $u\left(s_{i}, x_{j}, \omega_{s, y}\right)-u^{n, m}(r, x)$ and $\left(D_{s, y}^{M} u\right)(r, x)=u\left(r, x, \omega_{s, y}\right)-u(r, x)$ almost surely go to the same limit whatever $n$ and $m$ go to infinity or $r \uparrow s$ and $x \uparrow y$. By the theorem hypothesis this limit is $D^{-} u$.

Observe now that being $u$ bounded, and having $u^{n, m}$ the same bound, $D^{-} u$ and $u^{-}$ are also $L^{2}$-limits. So, using that $\delta^{M}$ is a closed operator, the left-hand side in (3.31) goes to $\delta^{M}\left(\left(u^{-}+D^{-} u\right) \cdot \mathbb{1}_{\mathbb{R}_{0}}\right)$ in $L^{2}$ if we prove that the terms on the right-hand side converge in $L^{2}$ to the limits defined by the proposition.

For the first term in the right-hand side, observe that $\delta^{M}$ coincides with the pathby-path integral because the integrand is deterministic. Then, using $u$ is bounded and the dominated convergence theorem we obtain the expected $L^{2}$-limit. For the second term, we have also a direct application of dominated convergence theorem.

In order to prove the nonbounded case observe that we can assume that $u$ is positive, because the formula that we want to prove is linear. Then, for the general case, we simply have to apply the result separately to the positive and negative parts.

So, let $u \geq 0$ and $u_{K}=u \wedge K$. Of course, $u_{K} \leq u$ and $u_{K}$ converges increasingly to $u$. We have, as a consequence of the first step, that

$$
\delta^{M}\left(\left(T^{-} u_{K}\right) \cdot \mathbb{1}_{\mathbb{R}_{0}}\right)=\int_{0}^{T} \int_{\mathbb{R}_{0}} u_{K}^{-}(s, x) N(d s, d x)-\int_{0}^{T} \int_{\mathbb{R}_{0}} T^{-} u_{K}(s, x) v(d x) d s .
$$

Being $u^{-}$and $T^{-} u$ in $L^{2}$, we have that $u_{K}^{-}$and $T^{-} u_{K}$ go up to $u^{-}$and $T^{-} u$ in $L^{2}$, respectively. So, hypothesis (3.26), the monotone convergence theorem and the closeness of the operator $\delta^{M}$ yield the result.

Remark 3.5. Notice from the proof of Proposition 3.4 that we can change the space $\mathbb{L}_{-}^{1,2}$ by a similar space with left limits with respect to the time variable $s$ but with right limits with respect the space variable $y$.

Remark 3.6. Observe that $T^{-} u=u^{-}$when $u$ is adapted to the filtration generated by $N$. Therefore, in such case

$$
\int_{0}^{t} \int_{\mathbb{R}_{0}} u^{-}(s, y) \widetilde{N}(d s, d y)=\delta^{M}\left(u^{-}(\cdot, \cdot) \mathbb{1}_{[0, t] \times \mathbb{R}_{0}}(\cdot, \cdot)\right)
$$

That is, in this case, the pathwise and Skorohod integrals with respect to $\widetilde{N}$ are the same. 
Observe that in the last two results there is no contribution of $W$ because on $\mathbb{R}_{0}$ the operator $\delta^{M}$ coincides with the Skorohod-type integral with respect to $\widetilde{N}$, as the following result shows.

Lemma 3.7. Let $\delta^{W}$ and $\delta^{N}$ be the adjoint operators of $D^{W}$ and $D^{N}$, respectively, and $u \in D o m \delta^{M}$. Then $u$ also belongs to Dom $\delta^{W} \cap \operatorname{Dom} \delta^{N}$ and

$$
\delta^{M}(u)=\sigma \delta^{W}(u \cdot, 0)+\delta^{N}\left(u \mathbb{1}_{\mathbb{R}_{0}}\right)
$$

Proof. This result is implied by (3.21) and (3.16).

\subsection{The anticipating Itô's formula}

The basic tool for our results is the following anticipative Itô formula. Recall that the process $X$ is introduced in (2.1) and $Y$ is the future average volatility, which is an anticipative process, even $\sigma$ is adapted.

Theorem 3.8. Let $\sigma^{2} \in \mathbb{L}^{1,2}$ and $F:[0, T] \times \mathbb{R} \times[0, \infty) \rightarrow \mathbb{R}$ be a bounded function in $C^{1,2,2}([0, T] \times$ $\mathbb{R} \times[0, \infty))$ with bounded derivatives. Then,

$$
\begin{aligned}
F\left(t, X_{t}, Y_{t}\right)-F\left(0, X_{0}, Y_{0}\right)= & \int_{0}^{t} \partial_{s} F\left(s, X_{s}, Y_{s}\right) d s+\int_{0}^{t} \partial_{x} F\left(s, X_{s}, Y_{s}\right)\left(r-\frac{\sigma_{s}^{2}}{2}-\lambda k\right) d s \\
& +\delta_{t}^{W, B}\left(\partial_{x} F\left(\cdot, X_{,}, Y_{.}\right) \sigma .\right)-\int_{0}^{t} \partial_{y} F\left(s, X_{s}, Y_{s}\right) \sigma_{s}^{2} d s \\
& +\rho \int_{0}^{t} \partial_{x y}^{2} F\left(s, X_{s}, Y_{s}\right) \Lambda_{s} d s+\frac{1}{2} \int_{0}^{t} \partial_{x x}^{2} F\left(s, X_{s}, Y_{s}\right) \sigma_{s}^{2} d s \\
& +\delta_{t}^{N}\left(T^{-} u \cdot \mathbb{1}_{\mathbb{R}_{0}}\right)+\int_{0}^{t} \int_{\mathbb{R}_{0}} T^{-} u(s, x) v(d x) d s
\end{aligned}
$$

where $\delta^{W, B}$ is the Skorohod integral with respect to the Brownian motion $\rho W_{s}+\sqrt{1-\rho^{2}} B_{s}, \Lambda_{s}:=$ $\left(\int_{s}^{T} D_{s}^{W} \sigma_{r}^{2} d r\right) \sigma_{s}$ and $u(s, x):=F\left(s, X_{s^{-}}+x, Y_{s}\right)-F\left(s, X_{s^{-}}, Y_{s}\right)$.

Proof. The proof is as in Alòs et al. [8] combined by Proposition 3.4 to treat the sum of jump terms.

We apply it to the random field $u(s, x)=F\left(s, X_{s_{-}}+x, Y_{s}\right)-F\left(s, X_{s_{-}}, Y_{s}\right)$. Here, the independence between $Z, B$, and $W$, the fact that $Y$ is a continuous process and the fact that $Z$ is a compound Poisson process with a finite number of jumps on every compact time interval play a key role.

Indeed, let $T_{i}$ denote these jump instants. Then,

$$
\begin{aligned}
& F\left(T_{i+1}, X_{T_{i+1}}, Y_{T_{i+1}}\right)-F\left(T_{i}, X_{T_{i}}, Y_{T_{i}}\right) \\
& \quad=\int_{T_{i}}^{T_{i+1}^{-1}} d F\left(s, X_{S}, Y_{S}\right)+F\left(T_{i+1}, X_{T_{i+1}}, Y_{T_{i+1}}\right)-F\left(T_{i+1}, X_{T_{i+1}^{-}}, Y_{T_{i+1}}\right) .
\end{aligned}
$$


The first term yields a standard Itô formula concerning continuous process, so Alòs et al. [8] results apply and we get the six first terms in the right-hand side of the Theorem 3.8 formula. On other hand, the sum of second terms is the path by path integral

$$
\int_{0}^{t} \int_{\mathbb{R}_{0}} u(s, x) N(d s, d x)
$$

Note here that $F, X_{-}, Y$ are left continuous so $u=u^{-}$. Then, using Proposition 3.4 we get the last sum is equal to:

$$
\delta_{t}^{N}\left(T^{-} u\right)+\int_{0}^{t} \int_{\mathbb{R}_{0}} T^{-} u(s, x) v(d x) d s
$$

\section{The Hull and White formula}

Now we have the following extension of the Hull and White formula.

Theorem 4.1. Let $\sigma$ and $X$ be as in Theorem 3.8. Then,

$$
\begin{aligned}
V_{t}= & E\left(B S\left(t, X_{t}, v_{t}\right) \mid \mathcal{F}_{t}\right)+\frac{\rho}{2} E\left(\int_{t}^{T} e^{-r(s-t)} \partial_{x} G\left(s, X_{s}, v_{s}\right) \Lambda_{s} d s \mid \mathcal{F}_{t}\right) \\
& +E\left(\int_{t}^{T} \int_{\mathbb{R}_{0}} e^{-r(s-t)}\left(T^{-} B S\left(s, X_{s-}+y, v_{s}\right)-T^{-} B S\left(s, X_{s-}, v_{s}\right) d s v(d y) \mid \mathcal{F}_{t}\right)\right. \\
& -\lambda k E\left(\int_{t}^{T} e^{-r(s-t)} \partial_{x} B S\left(s, X_{s}, v_{s}\right) d s \mid \mathcal{F}_{t}\right),
\end{aligned}
$$

where $G=\left(\partial_{x x}^{2}-\partial_{x}\right) B S$.

Remark 4.2. Note that in the case that $\sigma$ only depends on the filtration generated by $W$, we have $T^{-}=I d$. Consequently, in this case, we obtain the Hull and White formula given in Alos et al. [8].

Proof. This proof is similar to the one of the Theorem 4.2. in Alòs et al. [8]. Notice that $\mathrm{BS}\left(T, X_{T}, v_{T}\right)=V_{T}$. Then, from (2.2) we have

$$
e^{-r t} V_{t}=E\left(e^{-r T} \mathrm{BS}\left(T, X_{T}, v_{T}\right) \mid \mathcal{F}_{t}\right) .
$$

Now, our idea is to apply the Itô formula (Theorem 3.8) to the process $e^{-r t} \mathrm{BS}\left(t, X_{t}, v_{t}\right)$. As the derivatives of $\mathrm{BS}(t, x, \sigma)$ are not bounded we use an approximating argument, 
changing $v_{t}$ by

$$
v_{t}^{\epsilon}:=\sqrt{\frac{1}{T-t}\left(Y_{t}+\epsilon\right)},
$$

and $\mathrm{BS}(t, x, \sigma)$ by $\mathrm{BS}_{n}(t, x, \sigma):=\mathrm{BS}(t, x, \sigma) \psi_{n}(x)$, where $\psi_{n}\left(X_{t}\right):=\phi((1 / n) x)$, for some $\phi \in \mathcal{C}_{b}^{2}$ such that $\phi(x)=1$ for all $x<1$ and $\phi(x)=0$ for all $x>2$. Now, applying Theorem 3.8 between $t$ and $T$ to function

$$
F:(t, x, y) \longmapsto e^{-r t} \mathrm{BS}_{n}\left(t, x, \sqrt{\frac{y+\epsilon}{T-t}}\right)
$$

and grouping terms according to the type of derivative we obtain

$$
\begin{aligned}
& e^{-r T} \mathrm{BS}_{n}\left(T, X_{T}, v_{T}^{\epsilon}\right) \\
& =e^{-r t} \mathrm{BS}_{n}\left(t, X_{t}, v_{t}^{\epsilon}\right)+\int_{t}^{T} e^{-r s} \mathcal{L}_{\mathrm{BS}}\left(\sigma_{s}\right) \mathrm{BS}_{n}\left(s, X_{s}, v_{s}^{\epsilon}\right) d s \\
& -\frac{1}{2} \int_{t}^{T} e^{-r s} \partial_{\sigma} \mathrm{BS}_{n}\left(s, X_{s}, v_{s}^{\epsilon}\right) \frac{\left(\sigma_{s}^{2}-\left(v_{s}^{\epsilon}\right)^{2}\right)}{v_{s}^{\epsilon}(T-s)} d s-\lambda k \int_{t}^{T} e^{-r s} \partial_{x} \mathrm{BS}_{n}\left(s, X_{s}, v_{s}^{\epsilon}\right) d s \\
& +\delta^{W, B}\left(e^{-r s} \partial_{x} \mathrm{BS}_{n}\left(s, X_{s}, v_{s}^{\epsilon}\right) \sigma_{s} \mathbb{1}_{(t, T]}(s)\right)+\frac{\rho}{2} \int_{t}^{T} e^{-r s} \partial_{\sigma x}^{2} \mathrm{BS}_{n}\left(s, X_{s}, v_{s}^{\epsilon}\right) \frac{1}{v_{s}^{e}(T-s)} \Lambda_{s} d s \\
& +\delta_{t}^{N}\left(e^{-r s}\left(T^{-} \mathrm{BS}_{n}\left(s, X_{s_{-}}+y, v_{s}^{\epsilon}\right)-T^{-} \mathrm{BS}_{n}\left(s, X_{s^{-}}, v_{s}^{\epsilon}\right)\right)\right) \\
& +\int_{t}^{T} \int_{\mathbb{R}_{0}} e^{-r s}\left(T^{-} \mathrm{BS}_{n}\left(s, X_{s^{-}}+y, v_{s}^{\epsilon}\right)-T^{-} \mathrm{BS}_{n}\left(s, X_{s^{-}}, v_{s}^{\epsilon}\right)\right) d s v(d y) .
\end{aligned}
$$

Notice that $\mathcal{L}_{\mathrm{BS}}\left(\sigma_{s}\right) \mathrm{BS}_{n}\left(s, X_{s}, v_{s}^{e}\right)=\left(\mathcal{L}_{\mathrm{BS}}\left(\sigma_{s}\right) \mathrm{BS}\left(s, X_{s}, v_{s}^{e}\right)\right) \psi_{n}\left(X_{s}\right)+A_{n}(s)$, where

$$
\begin{aligned}
A_{n}(s)= & \frac{1}{2} \sigma_{s}^{2}\left[2 \partial_{x} \mathrm{BS}\left(s, X_{s}, v_{s}^{e}\right) \psi_{n}^{\prime}\left(X_{s}\right)+\mathrm{BS}\left(s, X_{s}, v_{s}^{\epsilon}\right)\left(\psi_{n}^{\prime \prime}\left(X_{s}\right)-\psi_{n}^{\prime}\left(X_{s}\right)\right)\right] \\
& +r \mathrm{BS}\left(s, X_{s}, v_{s}^{\epsilon}\right) \psi_{n}^{\prime}\left(X_{s}\right) .
\end{aligned}
$$

Also note that the classical relation between the Gamma, the Vega, and the Delta gives us that

$$
\partial_{\sigma} \mathrm{BS}(s, x, \sigma) \frac{1}{\sigma(T-s)}=\left(\partial_{x x}^{2}-\partial_{x}\right) \mathrm{BS}(s, x, \sigma)
$$


Elisa Alòs et al.

Then we can write

$$
\begin{aligned}
e^{-r T} \mathrm{BS}_{n} & \left(T, X_{T}, v_{T}^{\epsilon}\right) \\
= & e^{-r t} \mathrm{BS}_{n}\left(t, X_{t}, v_{t}^{\epsilon}\right)+\int_{t}^{T} e^{-r s}\left[\left(\mathcal{L}_{\mathrm{BS}}\left(\sigma_{s}\right) \mathrm{BS}\right)\left(s, X_{s}, v_{s}^{\epsilon}\right) \psi_{n}\left(X_{s}\right)+A_{n}(s)\right] d s \\
& -\frac{1}{2} \int_{t}^{T} e^{-r s}\left(\partial_{x x}^{2}-\partial_{x}\right) \mathrm{BS}\left(s, X_{s}, v_{s}^{e}\right) \psi_{n}\left(X_{s}\right)\left(\sigma_{s}^{2}-\left(v_{s}^{e}\right)^{2}\right) d s \\
& -\lambda k \int_{t}^{T} e^{-r s} \partial_{x} \mathrm{BS}_{n}\left(s, X_{s}, v_{s}^{\epsilon}\right) d s+\delta^{W, B}\left(e^{-r s} \partial_{x} \mathrm{BS}_{n}\left(s, X_{s}, v_{s}^{e}\right) \sigma_{s} \mathbb{1}_{(t, T]}(s)\right) \\
& +\frac{\rho}{2} \int_{t}^{T} e^{-r s}\left[\left(\partial_{x}\left(\partial_{x x}^{2}-\partial_{x}\right) \mathrm{BS}\right)\left(s, X_{s}, v_{s}^{\epsilon}\right) \psi_{n}\left(X_{s}\right)+\left(\partial_{x x}^{2}-\partial_{x}\right) \mathrm{BS}\left(s, X_{s}, v_{s}^{e}\right) \psi_{n}^{\prime}\left(X_{s}\right)\right] \Lambda_{s} d s \\
& +\delta_{t}^{N}\left(e^{-r s}\left(T^{-} \mathrm{BS}_{n}\left(s, X_{s-}+y, v_{s}^{e}\right)-T^{-} \mathrm{BS}_{n}\left(s, X_{s-}, v_{s}^{\epsilon}\right)\right)\right) \\
& +\int_{t}^{T} \int_{\mathbb{R}_{0}} e^{-r s}\left(T^{-} \mathrm{BS}_{n}\left(s, X_{s-}+y, v_{s}^{\epsilon}\right)-T^{-} \mathrm{BS}_{n}\left(s, X_{s-}, v_{s}^{\epsilon}\right)\right) d s v(d y) .
\end{aligned}
$$

Hence, taking into account that $\mathcal{L}_{\mathrm{BS}}\left(\sigma_{s}\right)=\mathcal{L}_{\mathrm{BS}}\left(v_{s}^{e}\right)+(1 / 2)\left(\sigma_{s}^{2}-\left(v_{s}^{e}\right)^{2}\right)\left(\partial_{x x}^{2}-\partial_{x}\right)$ it follows that (using the fact that $\mathcal{L}_{\mathrm{BS}}\left(v_{s}^{\epsilon}\right) \mathrm{BS}\left(s, X_{s}, v_{s}^{\epsilon}\right)=0$ )

$$
\begin{aligned}
& e^{-r T} \mathrm{BS}_{n}\left(T, X_{T}, v_{T}^{\epsilon}\right) \\
&=e^{-r t} \mathrm{BS}_{n}\left(t, X_{t}, v_{t}^{e}\right)+\int_{t}^{T} e^{-r s} A_{n}(s) d s \\
& \quad-\lambda k \int_{t}^{T} e^{-r s} \partial_{x} \mathrm{BS}_{n}\left(s, X_{s}, v_{s}^{\epsilon}\right) d s+\delta^{W, B}\left(e^{-r s} \partial_{x} \mathrm{BS}_{n}\left(s, X_{s}, v_{s}^{\epsilon}\right) \sigma_{s} \mathbb{1}_{(t, T]}(s)\right) \\
& \quad+\frac{\rho}{2} \int_{t}^{T} e^{-r s}\left[\left(\partial_{x}\left(\partial_{x x}^{2}-\partial_{x}\right) \mathrm{BS}\right)\left(s, X_{s}, v_{s}^{\epsilon}\right) \psi_{n}\left(X_{s}\right)+\left(\partial_{x x}^{2}-\partial_{x}\right) \mathrm{BS}\left(s, X_{s}, v_{s}^{e}\right) \psi_{n}^{\prime}\left(X_{s}\right)\right] \Lambda_{s} d s \\
&+\delta_{t}^{N}\left(e^{-r s}\left(T^{-} \mathrm{BS}_{n}\left(s, X_{s-}+y, v_{s}^{\epsilon}\right)-T^{-} \mathrm{BS}_{n}\left(s, X_{s-}, v_{s}^{\epsilon}\right)\right)\right) \\
&+\int_{t}^{T} \int_{\mathbb{R}_{0}} e^{-r s}\left(T^{-} \mathrm{BS}_{n}\left(s, X_{s-}+y, v_{s}^{\epsilon}\right)-T^{-} \mathrm{BS}_{n}\left(s, X_{s-}, v_{s}^{\epsilon}\right)\right) d s v(d y) .
\end{aligned}
$$

Now, taking conditional expectations we obtain that

$$
\begin{aligned}
E & \left(e^{-r T} \mathrm{BS}_{n}\left(T, X_{T}, v_{T}^{\epsilon}\right) \mid \mathcal{F}_{t}\right) \\
& =E\left\{e^{-r t} \mathrm{BS}_{n}\left(t, X_{t}, v_{t}^{\epsilon}\right)+\int_{t}^{T} e^{-r s} A_{n}(s) d s-\lambda k \int_{t}^{T} e^{-r s} \partial_{x} \mathrm{BS}_{n}\left(s, X_{s}, v_{s}^{\epsilon}\right) d s\right.
\end{aligned}
$$




$$
\begin{aligned}
& +\frac{\rho}{2} \int_{t}^{T} e^{-r s}\left[\left(\partial_{x}\left(\partial_{x x}^{2}-\partial_{x}\right) \mathrm{BS}\right)\left(s, X_{s}, v_{s}^{\epsilon}\right) \psi_{n}\left(X_{s}\right)+\left(\partial_{x x}^{2}-\partial_{x}\right) \operatorname{BS}\left(s, X_{s}, v_{s}^{\epsilon}\right) \psi_{n}^{\prime}\left(X_{s}\right)\right] \Lambda_{s} d s \\
& \left.+\int_{t}^{T} \int_{\mathbb{R}_{0}} e^{-r s}\left(T^{-} \mathrm{BS}_{n}\left(s, X_{s-}+y, v_{s}^{e}\right)-T^{-} \mathrm{BS}_{n}\left(s, X_{s-}, v_{s}^{\epsilon}\right)\right) d s v(d y) \mid \mathcal{F}_{t}\right\} .
\end{aligned}
$$

Let us remark that continuity of $\mathrm{BS}_{n}, v^{\epsilon}$ and left continuity of $X_{-}$imply that $\left(T^{-} \mathrm{BS}_{n}(S\right.$, $\left.X_{s^{-}}+y, v_{s}^{e}\right)=\mathrm{BS}_{n}\left(s, X_{s^{-}}+y, T^{-} v^{e}(s, y)\right)$.

Finally, we obtain the result proceeding as in the proof of Theorem 3 in Alòs et al. [8]. That is, letting first $n \uparrow \infty$, then $\epsilon \downarrow 0$ and using the dominated convergence theorem.

Remark 4.3. The additional term given by $T^{-} \mathrm{BS}$ can be detailed as follows. Suppose that $\sigma_{r}^{2}=$ $f\left(W_{u}, Z_{u}, u \leq r\right)$. Then we can define

$$
\widetilde{v}_{s}^{2}=\lim _{t \uparrow s, y \uparrow x} \frac{1}{T-t} \int_{t}^{T} T_{s, x}\left(\sigma_{r}^{2}\right) d r
$$

But for $r>s, T_{s, x}\left(\sigma_{r}^{2}\right)=f\left(W_{u}, Z_{u}+x \mathbb{1}_{\{s \leq u\}}, u \leq r\right)$,

$$
\tilde{v}_{s}^{2}=\lim _{t \uparrow s} \frac{1}{T-t} \int_{t}^{T} f\left(W_{u}, Z_{u}+x \mathbb{1}_{\{s \leq u\}}, u \leq r\right) d r=\frac{1}{T-s} \int_{s}^{T} \tilde{\sigma}_{r}^{2} d r
$$

where $\widetilde{\sigma}_{r}^{2}=f\left(W_{u}, Z_{u}+x \mathbb{1}_{\{s \leq u\}}, u \leq r\right)$.

For example, consider the following pure volatility jump case described in Álvarez [23]. See also Espinosa and Vives [24]. For $i \geq 0$, let $T_{i}$ be the jump instants and $\Delta_{T_{i}} Z$ be the jump sizes of process $Z$, with $T_{0}=0$. Assume that the dynamic of $\sigma$ is given by

$$
\sigma_{t}^{2}=\sum_{i=0}^{N_{t}} \sigma_{i}^{2} \mathbb{1}_{\left[T_{i}, T_{i+1}[\right.}(t),
$$

with $\sigma_{i}^{2}=\sigma_{i-1}^{2}+f\left(\Delta_{T_{i}} Z\right)$, for a certain function $f$. In this case, we have $\widetilde{\sigma}_{r}^{2}=\sigma_{r}^{2}+f(x) \mathbb{1}_{\{r \geq s\}}(r)$ and so, the explicit computation of $\widetilde{v}_{s}$ gives $\widetilde{v}_{s}^{2}=v_{s}^{2}+f(x)$.

\section{Short time behavior of the implied volatility}

In this section, we show that the short-time behavior of the at-the-money implied volatility is the same as in the case where the volatility $\sigma$ is independent of the filtration of $Z$, even the Hull and White formula is different in the last case (see Remark 4.2). This is a fact that must be taken in account for pricing and hedging.

Let $I_{t}\left(X_{t}\right)$ denote the implied volatility process. By definition it satisfies $V_{t}=$ $\mathrm{BS}\left(t, X_{t}, I_{t}\left(X_{t}\right)\right)$. Assume that $\sigma \in \mathbb{L}^{1,2}$ is as in model (2.1). Proceeding as in Alòs et al. [8], the derivative of the implied volatility with respect to the log-strike $k=\log K$ is

$$
\frac{\partial I_{t}}{\partial k}\left(x_{t}^{*}\right)=-\left.\frac{E\left(\int_{t}^{T}\left(\partial_{x} F\left(s, X_{s}, v_{s}\right)-(1 / 2) F\left(s, X_{s}, v_{s}\right)\right) d s \mid \mathscr{F}_{t}\right)}{\partial_{\sigma} \operatorname{BS}\left(t, x_{t}^{*}, I_{t}\left(x_{t}^{*}\right)\right)}\right|_{X_{t}=x_{t}^{*}}, \text { a.s., }
$$


where

$$
\begin{aligned}
F\left(s, X_{s}, v_{s}\right):= & \frac{\rho}{2} e^{-r(s-t)} \partial_{x} G\left(s, X_{s}, v_{s}\right) \Lambda_{s}-\lambda k e^{-r(s-t)} \partial_{x} \mathrm{BS}\left(s, X_{s}, v_{s}\right) \\
& +\int_{\mathbb{R}} e^{-r(s-t)}\left(\mathrm{BS}\left(s, X_{s-}+y, \widetilde{v}_{s}\right)-\operatorname{BS}\left(s, X_{s-}+y, \widetilde{v}_{s}\right)\right) v(d y) .
\end{aligned}
$$

Now, in order to study the limit of $\left(\partial I_{t} / \partial k\right)\left(x_{t}^{*}\right)$ as $T \downarrow t$ we need to introduce the following hypotheses:

(H1) $\sigma \in \mathbb{L}_{W}^{2,4}=L^{4}\left([0, T] \times \mathbb{R} ; \mathbb{D}_{W}^{2,4}\right)$.

(H2) There exists a constant $\alpha>-(1 / 2)$ such that, for all $0<t<s<r<T$,

$$
\begin{aligned}
E\left(\left(D_{s}^{W} \sigma_{r}\right)^{2} \mid \mathcal{F}_{t}\right) & \leq C(r-s)^{2 \alpha} \\
E\left(\left(D_{\theta}^{W} D_{s}^{W} \sigma_{r}\right)^{2} \mid \mathcal{F}_{t}\right) & \leq C(r-s)^{2 \alpha}(r-\theta)^{-2 \alpha} .
\end{aligned}
$$

(H3) For every fixed $t>0, \sup _{s, r, \theta \in[t, T]} E\left(\left(\sigma_{s} \sigma_{r}-\sigma_{\theta}^{2}\right)^{2} \mid \mathscr{F}_{t}\right) \rightarrow 0$ as $T \rightarrow t$.

Theorem 5.1. Under the Hypotheses (H1)-(H3) we have

(1) assume that $\alpha$ in (H2) is nonnegative and that there exists a $\boldsymbol{F}_{t}$-measurable random variable $D_{t}^{W,+} \sigma_{t}$ such that, for every $t>0$,

$$
\sup _{s, r \in[t, T]}\left|E\left(\left(D_{s}^{W} \sigma_{r}-D_{t}^{W,+} \sigma_{t}\right) \mid \mp_{t}\right)\right| \longrightarrow 0
$$

a.s. as $T \rightarrow t$; then

$$
\lim _{T \rightarrow t} \frac{\partial I_{t}}{\partial k}\left(x_{t}^{*}\right)=\frac{1}{\sigma_{t}}\left(\lambda k+\rho \frac{D_{t}^{W,+} \sigma_{t}}{2}\right),
$$

(2) assume that $\alpha$ in (H2) is negative and that there exists a $\boldsymbol{F}_{t}$-measurable random variable $L_{t}^{\alpha,+} \sigma_{t}$ such that, for every $t>0$,

$$
\frac{1}{(T-t)^{2+\alpha}} \int_{t}^{T} \int_{s}^{T} E\left(D_{s}^{W} \sigma_{r} \mid \mathscr{F}_{t}\right) d r d s-L_{t}^{\alpha,+} \sigma_{t} \longrightarrow 0
$$

a.s. as $T \rightarrow t$; then

$$
\lim _{T \rightarrow t}(T-t)^{-\alpha} \frac{\partial I_{t}}{\partial k}\left(x_{t}^{*}\right)=\frac{\rho}{\sigma_{t}} L_{t}^{\alpha,+} \sigma_{t}
$$


Proof. We can write

$$
\begin{aligned}
-\partial_{\sigma} \mathrm{BS}\left(t, x_{t}^{*}, I_{t}\left(x_{t}^{*}\right)\right) \frac{\partial I_{t}}{\partial k}\left(x_{t}^{*}\right) \\
=\left.\frac{\rho}{2} E\left(\int_{t}^{T} e^{-r(s-t)}\left(\partial_{x}-\frac{1}{2}\right) \partial_{x}\left(\partial_{x x}^{2}-\partial_{x}\right) \mathrm{BS}\left(s, X_{s}, v_{s}\right) \Lambda_{s} d s \mid \mathcal{F}_{t}\right)\right|_{X_{t}=x_{t}^{*}} \\
\quad+\left.E\left(\int_{t}^{T} \int_{\mathbb{R}} e^{-r(s-t)}\left(\partial_{x}-\frac{1}{2}\right)\left[\mathrm{BS}\left(s, X_{s}+y, \tilde{v}_{s}\right)-\mathrm{BS}\left(s, X_{s}, \tilde{v}_{s}\right)\right] v(d y) d s \mid \mathcal{F}_{t}\right)\right|_{X_{t}=x_{t}^{*}} \\
\quad-\left.\lambda k E\left(\int_{t}^{T} e^{-r(s-t)}\left(\partial_{x}-\frac{1}{2}\right) \partial_{x} \mathrm{BS}\left(s, X_{s}, v_{s}\right) d s \mid \mathcal{F}_{t}\right)\right|_{X_{t}=x_{t}^{*}}=T_{1}+T_{2}+T_{3} .
\end{aligned}
$$

The term $T_{2}$ is $O(T-t)$ due to the fact that the following majoration is uniform on $\sigma$

$$
|\mathrm{BS}(t, x, \sigma)|+\left|\partial_{x} \mathrm{BS}(t, x, \sigma)\right| \leq 2 e^{x}+K
$$

Now the result follows as in Alòs et al. [8, Proposition 6 and Theorem 7].

\section{Conclusion}

The Malliavin calculus for Lévy processes appears to be a natural tool to deal with the future average volatility in jump-diffusion models where the volatility is correlated with both Brownian motion and compound Poisson process driving the stock price process. Proceeding as in Alòs et al. [8], this powerful calculus allows us to identify the effect of both correlations. In particular, we have seen that the correlation with the asset price jumps has no effect on the short-time behavior of the volatility skew.

\section{Acknowledgments}

The authors would like to thank two anonymous referees for their useful comments and suggestions. The first author is supported by Grants MEC FEDER MTM 200606427 and SEJ2006-13537, the second author is partially supported by the CONACyT Grant 45684-F, and the last two authors are supported by Grant MEC FEDER MTM 200606427.

\section{References}

[1] A. L. Lewis, Option Valuation under Stochastic Volatility: With Mathematica Code, Finance Press, Newport Beach, Calif, USA, 2000.

[2] R. W. Lee, "Implied volatility: statics, dynamics, and probabilistic interpretation," in Recent Advances in Applied Probability, pp. 241-268, Springer, New York, NY, USA, 2005.

[3] A. Medvedev and O. Scaillet, "A simple calibration procedure of stochastic volatility models with jumps by short term asymptotics," Discussion Paper HEC 93, Genève and FAME, Université de Genève, Genève, Switzerland, 2004.

[4] D. Bates, "Jumps and stochastic volatility: exchange rate processes implicit in Deutsche Mark options," The Review of Financial Studies, vol. 9, no. 1, pp. 69-107, 1996. 
[5] O. E. Barndorff-Nielsen and N. Shephard, "Modelling by Lévy processes for financial econometrics," in Lévy Processes: Theory and Applications, pp. 283-318, Birkhäuser, Boston, Mass, USA, 2001.

[6] O. E. Barndorff-Nielsen and N. Shephard, "Econometric analysis of realized volatility and its use in estimating stochastic volatility models," Journal of the Royal Statistical Society. Series B, vol. 64, no. 2, pp. 253-280, 2002.

[7] P. Carr and L. Wu, "The finite moment log stable process and option pricing," The Journal of Finance, vol. 58, no. 2, pp. 753-778, 2003.

[8] E. Alòs, J. A. León, and J. Vives, "On the short-time behavior of the implied volatility for jumpdiffusion models with stochastic volatility," Finance and Stochastics, vol. 11, no. 4, pp. 571-589, 2007.

[9] E. Alòs, "A generalization of the Hull and White formula with applications to option pricing approximation," Finance and Stochastics, vol. 10, no. 3, pp. 353-365, 2006.

[10] J.-P. Fouque, G. Papanicolaou, and K. R. Sircar, Derivatives in Financial Markets with Stochastic Volatility, Cambridge University Press, Cambridge, UK, 2000.

[11] G. Bakshi, C. Cao, and Z. Chen, "Empirical performance of alternative option pricing models," The Journal of Finance, vol. 52, no. 5, pp. 2003-2049, 1997.

[12] D. Duffie, J. Pan, and K. Singleton, "Transform analysis and asset pricing for affine jump-diffusions," Econometrica, vol. 68, no. 6, pp. 1343-1376, 2000.

[13] J. L. Solé, F. Utzet, and J. Vives, "Canonical Lévy process and Malliavin calculus," Stochastic Processes and Their Applications, vol. 117, no. 2, pp. 165-187, 2007.

[14] A. Løkka, "Martingale representation of functionals of Lévy processes," Stochastic Analysis and Applications, vol. 22, no. 4, pp. 867-892, 2004.

[15] E. Petrou, "Malliavin calculus in Lévy spaces and applications to finance," Electronic Journal of Probability, vol. 13, no. 27, pp. 852-879, 2008.

[16] J. Gatheral, The Volatility Surface: A Practitioner's Guide, John Wiley \& Sons, New York, NY, USA, 2006.

[17] K.-I. Sato, Lévy Processes and Infinitely Divisible Distributions, vol. 68 of Cambridge Studies in Advanced Mathematics, Cambridge University Press, Cambridge, UK, 1999.

[18] D. Nualart and W. Schoutens, "Chaotic and predictable representations for Lévy processes," Stochastic Processes and Their Applications, vol. 90, no. 1, pp. 109-122, 2000.

[19] K. Itô, "Spectral type of the shift transformation of differential processes with stationary increments," Transactions of the American Mathematical Society, vol. 81, pp. 253-263, 1956.

[20] D. Nualart, The Malliavin Calculus and Related Topics, Probability and Its Applications, Springer, New York, NY, USA, 1995.

[21] E. Alòs, J. A. León, and J. Vives, "An anticipating Itô formula for Lévy processes," ALEA: Latin American Journal of Probability and Mathematical Statistics, vol. 4, pp. 285-305, 2008.

[22] G. Di Nunno, T. Meyer-Brandis, B. Øksendal, and F. Proske, "Malliavin calculus and anticipative Itô formulae for Lévy processes," Infinite Dimensional Analysis, Quantum Probability and Related Topics, vol. 8, no. 2, pp. 235-258, 2005.

[23] A. Álvarez, Modélisation de séries financières, estimation, ajustement de modèles et test d'hypothèses, M.S. thesis, Université Paul Sabatier Toulouse III, Toulouse, France, 2007.

[24] F. Espinosa and J. Vives, "A volatility-varying and jump-diffusion Merton type model of interest rate risk," Insurance: Mathematics E Economics, vol. 38, no. 1, pp. 157-166, 2006. 


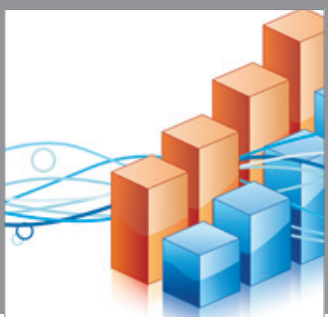

Advances in

Operations Research

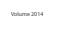

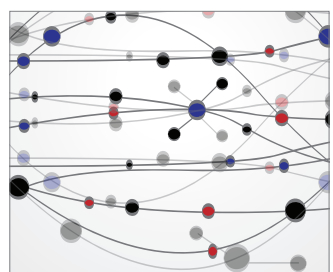

\section{The Scientific} World Journal
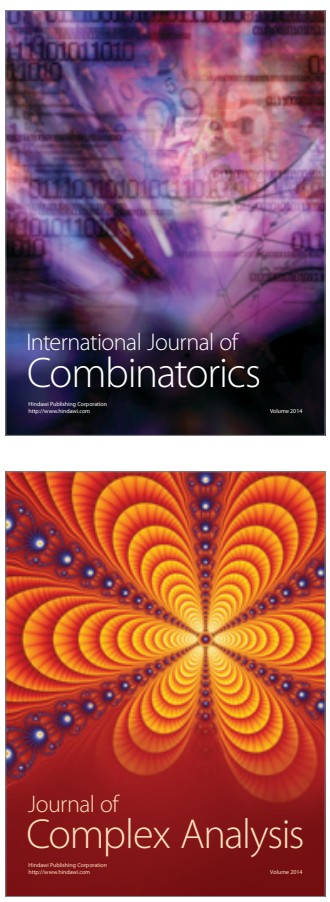

International Journal of

Mathematics and

Mathematical

Sciences
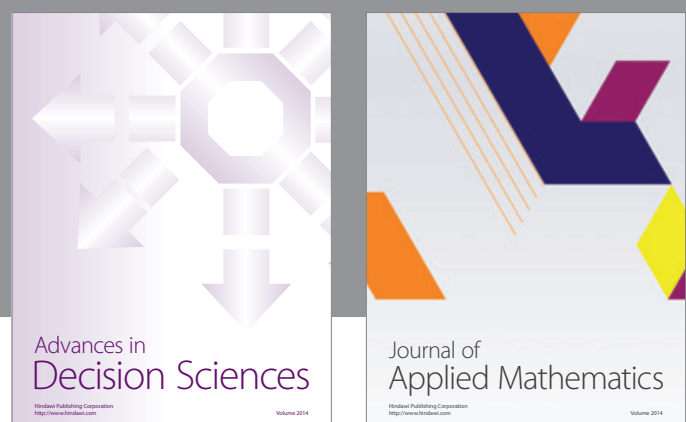

Journal of

Applied Mathematics
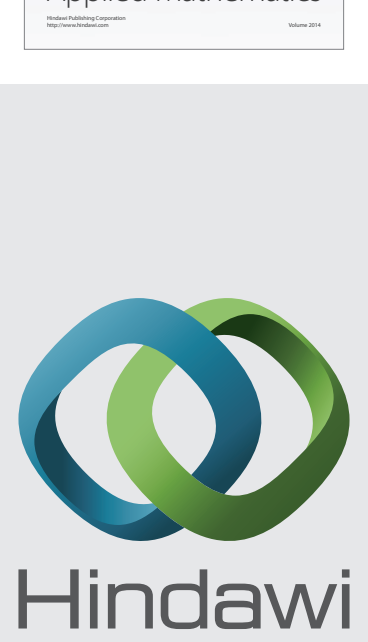

Submit your manuscripts at http://www.hindawi.com
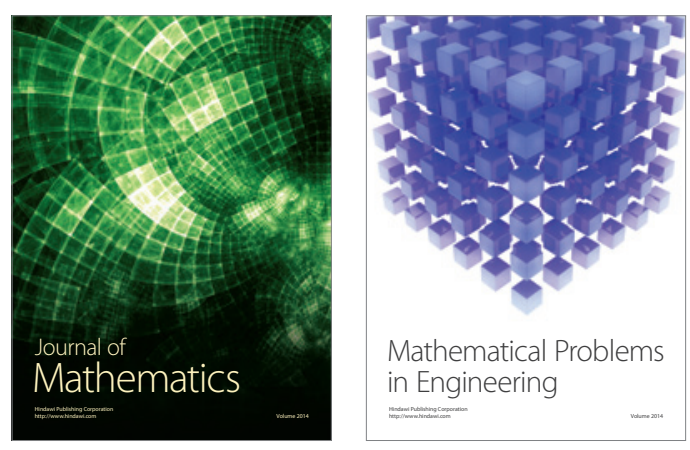

Mathematical Problems in Engineering
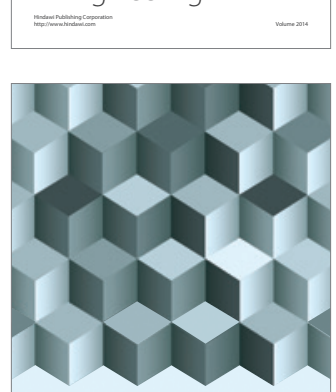

Journal of

Function Spaces
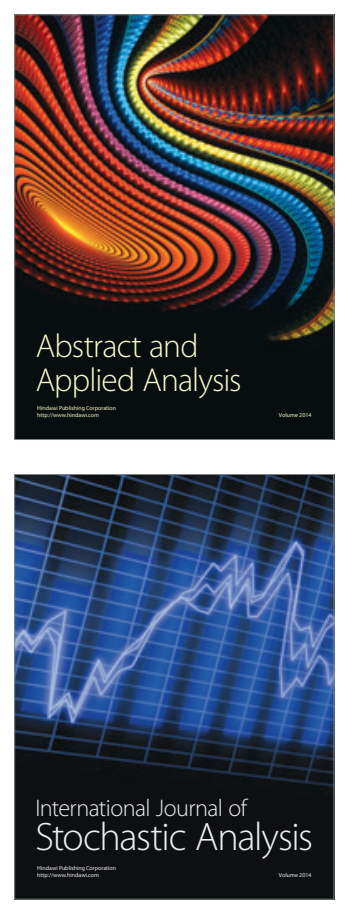

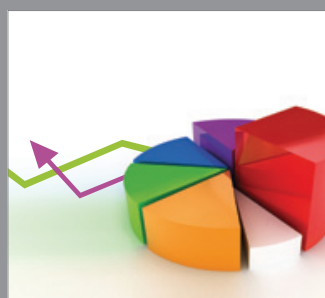

ournal of

Probability and Statistics

Promensencen
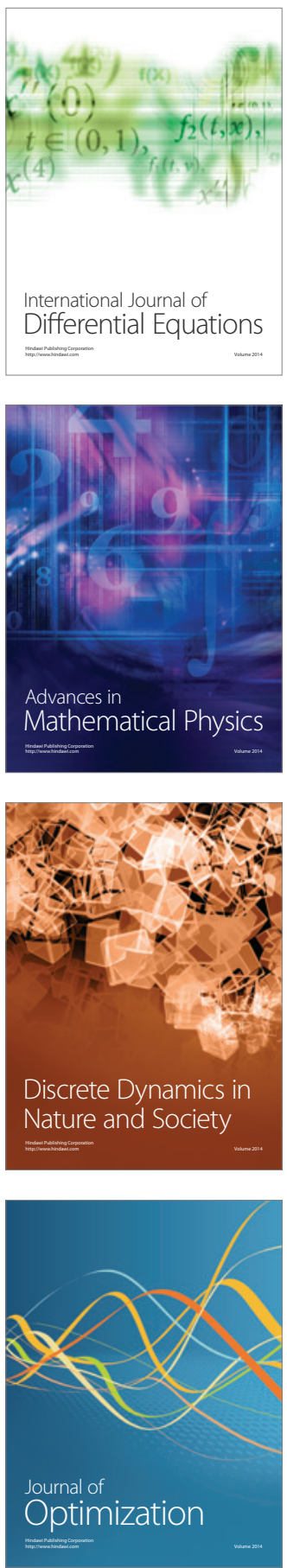